\title{
Article \\ Postural Stability in Single-Leg Quiet Stance in Highly Trained Athletes: Sex and Sport Differences
}

\author{
Nebojša Trajković ${ }^{1}$, Darjan Smajla ${ }^{2,3}$, Žiga Kozinc ${ }^{2,4}$ (D) and Nejc Šarabon ${ }^{2,3,4,5, *(\mathbb{D})}$ \\ 1 Faculty of Sport and Physical Education, University of Niš, Čarnojevićeva 10a, 18000 Nis, Serbia; \\ nele_trajce@yahoo.com \\ 2 Faculty of Health Sciences, University of Primorska, Polje 42, SI-6310 Izola, Slovenia; \\ darjan.smajla@fvz.upr.si (D.S.); ziga.kozinc@fvz.upr.si (Ž.K.) \\ 3 Human Health Department, InnoRenew CoE, Livade 6, SI-6310 Izola, Slovenia \\ 4 Andrej Marušič Institute, University of Primorska, Muzejski trg 2, SI-6000 Koper, Slovenia \\ 5 Laboratory for Motor Control and Motor Behavior, S2P, Science to Practice, Ltd., Tehnološki Park 19, \\ SI-1000 Ljubljana, Slovenia \\ * Correspondence: nejc.sarabon@fvz.upr.si
}

check for updates

Citation: Trajković, N.; Smajla, D.;

Kozinc, Ž.; Šarabon, N. Postural Stability in Single-Leg Quiet Stance in Highly Trained Athletes: Sex and Sport Differences. J. Clin. Med. 2022, 11, 1009. https://doi.org/ $10.3390 /$ jcm 11041009

Academic Editors: David Rodríguez-Sanz and Naama W Constantini

Received: 11 January 2022

Accepted: 15 February 2022

Published: 15 February 2022

Publisher's Note: MDPI stays neutral with regard to jurisdictional claims in published maps and institutional affiliations.

Copyright: (C) 2022 by the authors. Licensee MDPI, Basel, Switzerland. This article is an open access article distributed under the terms and conditions of the Creative Commons Attribution (CC BY) license (https:// creativecommons.org/licenses/by/ $4.0 /)$.

\begin{abstract}
This study aimed to determine if there is a difference in postural stability in highly trained adolescents and young adult athletes regarding sex and sport. The participants were young athletes ( $n=464)$ from seven different sports. We considered the center of pressure (CoP) velocity (total, anterior-posterior (AP) and medial-lateral (ML)), CoP amplitude (AP and ML), and CoP frequency (AP and ML), as assessed by single-leg quiet stance test. Significant interactions were found between sex and sport for all CoP variables $(p<0.02)$. Additionally, a significant main effect of sport was also found in all CoP variables $(p=0.01)$. Regarding sex, significant effects were found for all CoP amplitude variables $(p=0.01)$, as well as for CoP velocity variables, except for CoP ML $(p=0.06)$. Moreover, there was no sex effect for CoP frequency AP $(p=0.18)$. The results of the current study confirm the claim that the criteria for optimal postural strategies for elite athletes likely depend on a given sport.
\end{abstract}

Keywords: postural sway; balance; equilibrium; elite athletes; gender effect

\section{Introduction}

Postural stability is considered a very important factor for athletes in different sports [1] Due to its potential role in mitigating risk for injuries, postural stability has been the subject of interest of researchers. Postural stability evaluated through assessment of body sway enables quantifying the function of maintaining equilibrium during periods of standing still, locomotion, and any activities requiring a high degree of balance performance [2]. Evidence from a systematic review [3] suggests that athletes sway less than nonathletes and that highly trained elite athletes sway less than low-level athletes. The importance of good stability in some sports, (i.e., ballet, dance, gymnastics) is obvious. Previous comparisons of body sway among athletes from different sports have shown that gymnasts have better postural stability than football players, swimmers, and basketball players [4,5]. Negahban et al. [6] suggested that elite athletes may be more efficient in conditions consistent with their main experience and process of training. Accordingly, recent evidence suggests that sport-specific expertise induces alterations in sensory integration that underpins spatial referencing and postural control [7].

Novel research indicates that postural stability in athletes is not influenced by sex [8]. However, some studies noted better postural stability in females, which was suggested to be related to earlier physical and psychological maturation processes [9] and superior sensory integration [10]. Previous research has documented that female athletes have different anatomical characteristics, which could explain lower postural sway [11]. The development 
of proprioception and vestibular functions in females is also one factor that could interact with the improvement of the postural stability system [12]. These sex differences and inconsistency during childhood and adulthood were confirmed in several studies [13-16], indicating that girls tend to have better postural stability during childhood, while during adulthood, the situation is reversed. Therefore, improving the understanding of sportspecific patterns in postural stability and its interaction with sex is important, in order to develop better injury prevention programs and decrease injury risk.

Single-leg body sway parameters can be used for analyzing the static performance of stabilization in the condition of unilateral distribution of body weight, which is usual in sports activities. Good single-leg stabilization characteristic reflects on the smaller increase in vertical force and the shorter weight transfer in different movement tasks [17,18]. The single-leg stance test is also recommended for clinicians as a useful tool for a brief assessment of the risk of falling [19]. Therefore, evaluation of postural sway in single-leg tests presents an important stability evaluation tool. The importance of postural stability in sport and everyday life has been well recognized and confirmed. However, only a few studies investigated postural stability considering sport and sex using different tests $[20,21]$. Moreover, most studies were conducted on children or older adults $[9,11-13,15,17]$. Having in mind that single-leg stance measurement has more applications in clinical and sport medicine settings [22] and that most injurious falls occurred in activities that involved single-leg stance [23], it is of great importance to understand the possible sport-specific characteristics of postural stability. Accordingly, there is a widespread call to identify a postural stability measure that can best distinguish between different sports and sex in highly trained young athletes.

In light of the aforementioned evidence, we used a previously collected database containing more than 400 participants, who all performed single-leg body sway assessments with open eyes. We chose the single-leg stance test because of its similarity with the movements in sports that require balancing on a single leg and the fact that athletes must be able to maintain good postural stability before any kind of motor action, in order to act efficiently [24]. The aim of the study was to assess the postural stability during single-leg quiet stance in highly trained male and female young athletes from different sports. We expected to observe differences in center of pressure (CoP) characteristics between sex and sports in highly trained young athletes.

\section{Materials and Methods}

\subsection{Participants}

The participants in the present study were young athletes $(n=464)$ from 7 different sports. The sample was taken from the database of a larger project, exploring interlimb asymmetries and performance in athletes $[25,26]$. All sports groups that performed postural sway assessments and involved both male and female participants were considered. Details of the sample sizes for each group, along with baseline demographic data, are presented in Table 1. Exclusion criteria included lower leg injuries in the past 6 months and possible neurological or noncommunicable diseases self-reported by participants. Participants were given detailed information about the testing procedures and were required to sign a written informed consent form prior to the measurements. For minor participants, parents or guardians were also notified and signed an informed consent form on their behalf. The National Committee for Medical Ethics of the Republic of Slovenia approved the experimental protocol (Approval Number 0120-99/2018/5) and was conducted in accordance with the latest revision of the Declaration of Helsinki.

\subsection{Procedures}

Body sway was assessed in a single-leg stance position without footwear. Participants performed three $30 \mathrm{~s}$ repetitions with each leg in the single-leg position, with $60 \mathrm{~s}$ long breaks between repetitions. The experimenter began the acquisition after stabilization (1-2 s). The postural sway was analyzed on the preferred leg, which was determined 
as the leg that the participant would use to kick a ball. The hip of the opposite leg (i.e., non-standing leg) was in a neutral position $\left(0^{\circ}\right)$, and the thigh was parallel to the standing leg, while the knee was flexed at $90^{\circ}$ and was not allowed to touch the standing leg. The standing leg's knee was in the extended position but not hyperextended (locked). The hands were placed on the hips.

Table 1. Basic participant data.

\begin{tabular}{|c|c|c|c|c|c|c|}
\hline & $n$ & Age (years) & Body Height (cm) & Body Mass (kg) & Weekly Training & Years of Training \\
\hline Basketball-M & 107 & $17.4(2.2)$ & $189.3(8.2)$ & $81.4(12.9)$ & $6.4(1.9)$ & $6.9(2.4)$ \\
\hline Basketball-F & 58 & 16.7 (1.6) & $175.2(5.6)$ & $70.2(11.2)$ & $5.5(1.3)$ & $6.4(2.5)$ \\
\hline Dance-M & 23 & $24.2(5.9)$ & $179.0(4.9)$ & $71.7(6.6)$ & $5.9(2.2)$ & $12.0(4.4)$ \\
\hline Dance-F & 54 & $22.3(7.0)$ & $166.9(5.3)$ & $55.3(6.1)$ & $6.6(2.6)$ & $9.9(4.0)$ \\
\hline $\begin{array}{l}\text { Track and } \\
\text { Field-M }\end{array}$ & 21 & $17.8(2.6)$ & $180.5(5.8)$ & 73.8 (7.9) & $5.4(1.6)$ & $6.5(3.1)$ \\
\hline $\begin{array}{l}\text { Track and } \\
\text { Field-M }\end{array}$ & 8 & $17.7(3.0)$ & $167.2(3.7)$ & $60.3(5.8)$ & $5.4(1.1)$ & $6.3(2.2)$ \\
\hline Running-M & 31 & $29.2(8.8)$ & $181.2(5.6)$ & $77.2(6.8)$ & $5.2(2.5)$ & $11.0(8.8)$ \\
\hline Running-F & 18 & $36.9(10.9)$ & $166.0(8.1)$ & $60.9(7.6)$ & $4.0(1.7)$ & $7.7(4.5)$ \\
\hline Tennis-M & 68 & $17.2(10.4)$ & $175.0(11.1)$ & $65.2(12.1)$ & $6.1(2.8)$ & $8.9(3.6)$ \\
\hline Tennis-F & 42 & $15.9(3.0)$ & $168.5(8.4)$ & $60.0(9.9)$ & $6.3(3.2)$ & $8.2(3.9)$ \\
\hline Martial arts-M & 18 & $19.9(3.1)$ & $180.3(6.0)$ & $75.5(8.9)$ & $5.6(1.3)$ & $7.7(2.5)$ \\
\hline Martial arts-F & 17 & $19.7(3.4)$ & $169.1(6.6)$ & $60.1(5.1)$ & $5.1(1.4)$ & $7.7(2.8)$ \\
\hline $\begin{array}{c}\text { Speed } \\
\text { skating-M }\end{array}$ & 12 & $16.8(5.1)$ & 169.5 (15.5) & $61.3(16.5)$ & $5.3(1.9)$ & $6.9(3.4)$ \\
\hline $\begin{array}{c}\text { Speed } \\
\text { skating-M }\end{array}$ & 7 & $16.9(3.4)$ & $161.1(8.4)$ & $57.3(10.9)$ & $4.9(2.0)$ & $6.0(3.9)$ \\
\hline
\end{tabular}

M-male; F-female.

A piezoelectric platform (model 9260AA, Kistler, Winterthur, Switzerland) was used to acquire ground reaction force data at a sampling rate of $1000 \mathrm{~Hz}$. The data were automatically filtered (low-pass Butterworth, 2nd order, $10 \mathrm{~Hz}$ ) in the software MARS (version 4.0, Kistler, Winterthur, Switzerland). Additionally, data were automatically processed in MARS to obtain outcome variables of interest. For further analysis, an average of three replicates was used for all outcome variables. We considered mean CoP velocity (total, anterior-posterior (AP) and medial-lateral (ML)), CoP amplitude (AP and ML) and $\mathrm{CoP}$ frequency (AP and ML). $\mathrm{CoP}$ amplitude was determined as the average $\mathrm{CoP}$ sway in the AP or ML direction, calculated as the total length of the COP sway path in a given direction only, divided by the number of directional changes. CoP frequency was defined as the frequency of $\mathrm{CoP}$ oscillations, calculated as the number of peaks in the $\mathrm{AP}$ or ML direction (i.e., changes in the direction of CoP motion) divided by the measurement time [27].

\subsection{Statistical Analysis}

Statistical analysis was performed in SPSS (version 25.0; SPSS Inc., Chicago, IL, USA). Descriptive statistics were calculated and reported as mean \pm standard deviation. The normality of the data distribution was checked with Shapiro-Wilk tests $(p \leq 0.121)$. A $2 \times 7$ MANCOVA was used to examine the interaction effect between sex and sport on a multivariate level. We used a $2 \times 7$ ANCOVA (between-subject design) to evaluate the sex and sports effects on body sway measures after controlling their effect for age (mean centered), body height (mean centered), and BMI (mean centered). The main effects of sex and sports estimated mean differences between men and women, and various sports players, respectively. The sex $\times$ sport interaction effect was employed to determine whether various sports players on average differ in body sway measures depending on the sex of sports players. For comparison of the sports included, the post hoc test was used. The effect sizes (ES) pertaining to ANOVA were expressed as partial eta squared ( $\eta 2)$ and interpreted as small $(<0.13)$, medium $(0.13-0.26)$, and large $(>0.26)$ [28]. 


\section{Results}

CoP variables, velocity, amplitude and frequency for preferred leg by sex and sports are presented in Table 2. Figure 1 represents the data for both sexes combined. A $2 \times 7$ MANCOVA showed that all studied variables significantly depend on sex $(\mathrm{F}=5.936$, $p=0.001, \eta 2=0.087)$, sport $(\mathrm{F}=14.614, p=0.001, \eta 2=0.185)$, and sport $\times$ sex interaction $(\mathrm{F}=2.561, p=0.001, \eta 2=0.039)$. A $2 \times 7$ ANCOVA followed and showed significant interaction between sex and sport for all CoP velocity variables $(p=0.01)$ with small effect size $(\eta 2=0.046-0.073)$. There were significant main effects for sex $(p<0.001)$ and sport participation $(p<0.001)$. Concerning sex, females reported lower scores for CoP velocity than males $(p=0.01 ; \eta 2=0.027-0.079$, small ES), except for CoP velocity ML, where no significant effect of sex was found ( $p=0.06 ; \eta 2=0.08$, small ES). Similarly, there was a significant effect of sports $(p=0.01)$, with small ES ranging from $\eta 2=0.062$ to $\eta 2=0.093$.

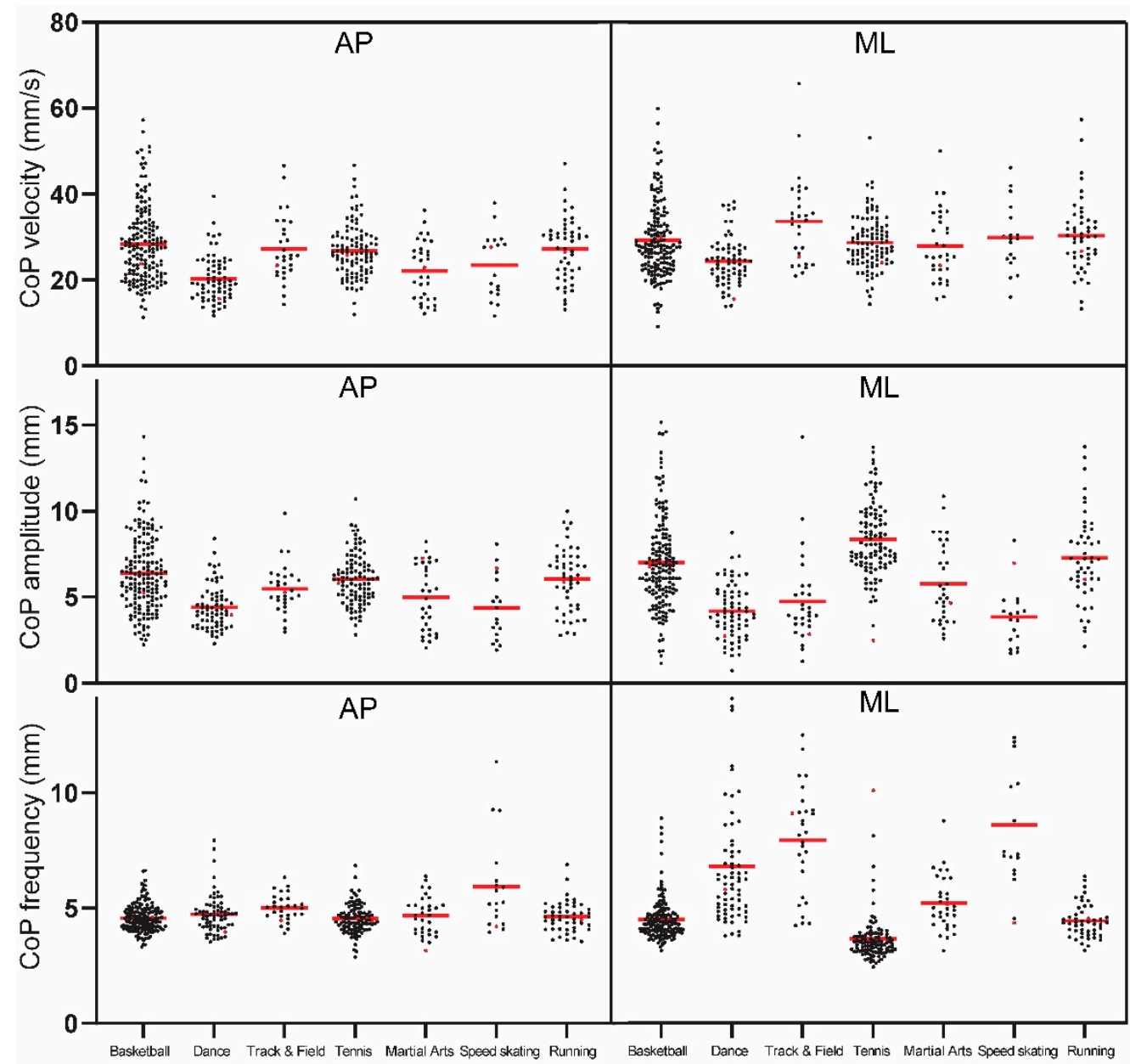

Figure 1. Representation of body sway data across sports (anterior-posterior direction, left panel; medial-lateral direction, right panel). Horizontal lines represent the mean of the groups). Statistical values are included in Table 2.

There were significant sex $\times$ sport interactions found for CoP frequency (CoP AP $\mathrm{F}=4.51 ; p=0.01 ; \mathrm{CoP} \mathrm{ML} \mathrm{F}=2.45 ; p=0.02)$. Moreover, there was no main effect of sex for CoP AP frequency $(p>0.05)$. Regarding sport modality, there was a significant main effect for CoP AP and ML frequency ( $p=0.01 ; \eta 2=0.143-0.400)$. 
Table 2. Comparison of CoP velocity, amplitude, and frequency according to sex and sport; values are mean \pm SD. Statistically significant effect are in bold.

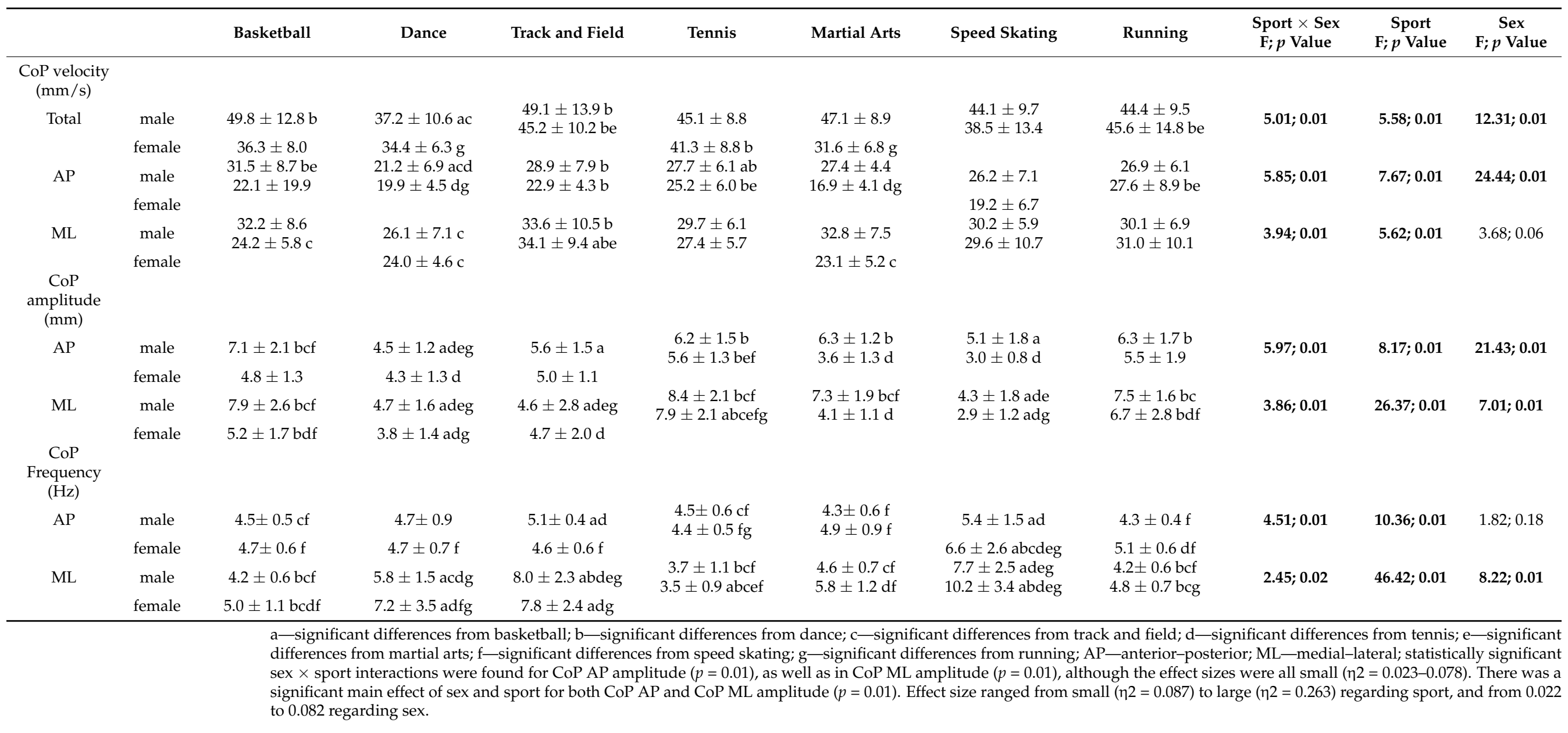




\section{Discussion}

This study aimed to analyze characteristics of single-leg quiet stance body sway in a highly trained athletic population and to explore the effects of sex and sport. The major findings in the current study point to sport-specific characteristics regarding postural stability in single-leg stance. Results for CoP velocity and amplitude clearly show that dancers are better able to maintain a stable single-leg stance than athletes from other sports. Moreover, the results of the present study show that, when comparing highly trained athletes of both sexes, there were differences in almost all $\mathrm{CoP}$ variables. Male athletes presented higher values of $\mathrm{CoP}$ velocity and amplitude but also for $\mathrm{CoP}$ frequency during single-leg stance.

The single-leg upright stance represents a challenging part of human locomotion because, compared with bipedal stance, it requires keeping the center of body mass within the smaller area of the support [13], which leads to more corrective movements by the postural control system in order to maintain balance [29]. Female athletes in the current study reported lower scores $(p=0.01)$ for CoP velocity $(\eta 2=0.030-0.079)$ and amplitude ( $\eta 2=0.055-0.082)$, compared with male athletes. Studies investigating postural control between sexes showed inconsistent results depending on the age group. Boys aged nine years showed significantly poorer single-leg postural stability than the girls of the same age [13], and similar was for participants in adolescent age [14]. On the contrary, female young adults seem to have lower postural stability, as shown by higher $\mathrm{CoP}$ velocities, compared with males [16]. The differences remain through adulthood as well in older adults-women tend to be less stable than men during single stance [15]. The main predictor that could influence this inconsistency in the results is the visual system, which was found to be the primary sensory system involved in maintaining postural stability in a broad range of age groups [30]. One more factor that could change the variability in postural stability is physical activity. This was confirmed in a study conducted on healthy young adults, in which no differences between sexes were detected when participants were physically active [31]. Female athletes in the current study showed better postural stability, compared with males with the difference being most pronounced for CoP velocity and amplitude, while the difference in $\mathrm{CoP}$ frequency was noted in the medial-lateral direction only $(p<0.05)$. Reduced CoP sway area and velocity in females of similar age were also noted in the recent study [21]. Possible reasons for better postural stability in this age are the maturation process [32] lower body weight [13], as well as better proprioception and control due to smaller absolute muscle mass and strength [33]. Additionally, it was stated that sex differences exist in children and adolescents due to the significantly lower body height in girls [13]. However, the participants in the abovementioned study were untrained children that were younger than participants in the current study, which may account for the discrepancies in some studies. Therefore, reasons for better postural control in younger age, as well as the reasons for the decline in later ages, should be investigated further.

It was stated that the postural balance of elite athletes should be always monitored, due to the establishment of sport-specific imbalances that could affect their performance [34]. The results of the current study suggest that dancers have better postural stability during single-leg stance than athletes from other sports, in all measured CoP characteristics. The differences are probably the result of adaptive balance strategies used by dancers in training, in which both abilities, cognitive and physical, are coordinated [35]. Additionally, their continuous training that uses balance control could minimize the effect of external perturbations [36] and thus improve postural control. However, the increase in body sway in the absence of vision in ballet dancers was previously reported by Bruyneel et al. [37]. Matsuda et al. [20] showed that soccer players make greater use of the somatosensory system during single-leg stance, compared with basketball players, swimmers, and nonathletes. However, highly trained female volleyball players showed higher $\mathrm{CoP}$ fractal dimensions, compared with controls, which is probably due to the adoption of certain habits. [38]. According to the authors, these high values show evidence for flexible and variable strategies of maintaining balance by highly trained athletes. This was confirmed in the current 
study among young highly trained athletes in that dancers were better able to maintain a stable, single-leg stance, compared with athletes from other sports. Only martial arts showed similar results to those of dance for CoP velocity and amplitude but also higher. The mechanism behind the best postural stability in dancers may be associated with the development of a motor skill for voluntary stabilization of important muscle groups, as well as better sensorimotor solutions for posture control [39]. The importance of dance exercise in maintaining good postural stability was well documented in adolescent females [40].

Some limitations of the present study need to be acknowledged. Across sports, the sample sizes varied considerably and were relatively small for some of the sports. Moreover, the samples were not sex balanced (e.g., dancers. Despite taking sexes into account as a factor in analyses, some main effects could still be driven by a larger representation of one sex in the sample (e.g., females in dancing). However, having in mind that, in some sports, it is hard to find highly trained athletes on an elite level, and since we included a considerable number of different sports, it was of great importance to conduct and analyze body sway because of important clinical implications in young athletes. Moreover, we did not include a healthy control group, which could have strengthened our interpretations. One more limitation is the fact that measures were assessed only during static conditions. Most of the selected sports rely more on dynamic conditions demands than static postures. Therefore, future studies should use both dynamic and static postural tests, in order to provide an overall assessment of balance in different sports. Nevertheless, the greatest strength of this study is encompassing a large number of sports and highly trained athletes compared at this age.

\section{Conclusions}

According to our findings, postural stability in highly trained adolescents and young adult athletes was influenced by sex and sport. Female athletes showed better postural stability than male athletes. The athletes engaged in dance showed the highest postural control, compared with other sports. The results of the current study confirm the claim that the criteria for optimal postural strategies for elite athletes likely depend on a given sport. This is of great importance in providing additional information about postural control abilities in highly trained athletes from different sports.

Author Contributions: Conceptualization, all authors; methodology, N.Š.; software, N.Š.; validation, N.Š. and D.S.; formal analysis, N.T.; investigation, Ž.K. and D.S.; resources, N.Š.; data curation, N.T., D.S. and Ž.K.; writing—original draft preparation, N.T. and Ž.K.; writing-review and editing, D.S. and N.Š.; visualization, all authors.; supervision, N.Š.; project administration, D.S. and N.Š.; funding acquisition, N.Š. All authors have read and agreed to the published version of the manuscript.

Funding: This study was supported by the Slovenian Research Agency through a project entitled "Body asymmetries as a risk factor in musculoskeletal injury development: studying aetiological mechanisms and designing corrective interventions for primary and tertiary preventive care" (TELASI-PREVENT (L5-1845)). The agency played no role in the conceptualization of the study, data acquisition, data analysis, or manuscript writing. Žiga Kozinc acknowledges the support of the Rectors Fund of the University of Primorska (internal post-doctoral project RAVOTEZ: Assessing Balance with Quantifying Transient Behavior of Postural Sway: From Validation To Practical Application; Grant Number: 2991-3-2/21). The fund played no role in the conceptualization of the study, data acquisition, data analysis, or manuscript writing.

Institutional Review Board Statement: The National Committee for Medical Ethics of the Republic of Slovenia approved the experimental protocol (Approval Number 0120-99/2018/5). The protocol was conducted in accordance with the latest revision of the Declaration of Helsinki.

Informed Consent Statement: Informed consent was obtained from all subjects involved in the study.

Data Availability Statement: The data presented in this study are available on request from the corresponding author.

Conflicts of Interest: The authors declare no conflict of interest. 


\section{References}

1. Hrysomallis, C. Balance Ability and Athletic Performance. Sports Med. 2011, 41, 221-232. [CrossRef] [PubMed]

2. Assaiante, C.; Mallau, S.; Viel, S.; Jover, M.; Schmitz, C. Development of postural control in healthy children: A functional approach. Neural Plast. 2005, 12, 109-118. [CrossRef] [PubMed]

3. Kiers, H.; Van Dieën, J.; Dekkers, H.; Wittink, H.; Vanhees, L. A systematic review of the relationship between physical activities in sports or daily life and postural sway in upright stance. Sports Med. 2013, 43, 1171-1189. [CrossRef] [PubMed]

4. Bressel, E.; Yonker, J.C.; Kras, J.; Heath, E.M. Comparison of static and dynamic balance in female collegiate soccer, basketball, and gymnastics athletes. J. Athl. Train. 2007, 42, 42.

5. Davlin, C.D. Dynamic balance in high level athletes. Percept. Mot. Skills 2004, 98, 1171-1176. [CrossRef]

6. Negahban, H.; Aryan, N.; Mazaheri, M.; Norasteh, A.A.; Sanjari, M.A. Effect of expertise in shooting and Taekwondo on bipedal and unipedal postural control isolated or concurrent with a reaction-time task. Gait Posture 2013, 38, 226-230. [CrossRef]

7. Thalassinos, M.; Fotiadis, G.; Arabatzi, F.; Isableu, B.; Hatzitaki, V. Sport Skill—Specific expertise biases sensory integration for spatial referencing and postural control. J. Mot. Behav. 2018, 50, 426-435. [CrossRef]

8. Jastrzębska, A.D. Gender differences in postural stability among 13-year-old alpine skiers. Int. J. Environ. Res. Public Health 2020, 17, 3859. [CrossRef]

9. Butz, S.M.; Sweeney, J.K.; Roberts, P.L.; Rauh, M.J. Relationships among age, gender, anthropometric characteristics, and dynamic balance in children 5 to 12 years old. Pediatr. Phys. Ther. 2015, 27, 126-133. [CrossRef]

10. Steindl, R.; Kunz, K.; Schrott-Fischer, A.; Scholtz, A.W. Effect of age and sex on maturation of sensory systems and balance control. Dev. Med. Child Neurol. 2006, 48, 477-482. [CrossRef]

11. Dorneles, P.P.; Pranke, G.I.; Mota, C.B. Comparison of postural balance between female and male adolescents. Fisioter. Pesq. 2013, 20, 210-214. [CrossRef]

12. Smith, A.; Ulmer, F.; Wong, D. Gender differences in postural stability among children. J. Hum. Kinet. 2012, 33, 25-32. [CrossRef] [PubMed]

13. Lee, A.J.Y.; Lin, W.-H. The influence of gender and somatotype on single-leg upright standing postural stability in children. J. Appl. Biomech. 2007, 23, 173-179. [CrossRef] [PubMed]

14. Schedler, S.; Kiss, R.; Muehlbauer, T. Age and sex differences in human balance performance from 6-18 years of age: A systematic review and meta-analysis. PLoS ONE 2019, 14, e0214434. [CrossRef] [PubMed]

15. Riva, D.; Mamo, C.; Fanì, M.; Saccavino, P.; Rocca, F.; Momenté, M.; Fratta, M. Single stance stability and proprioceptive control in older adults living at home: Gender and age differences. J. Aging Res. 2013, 2013, 561695. [CrossRef] [PubMed]

16. Błaszczyk, J.W.; Beck, M.; Sadowska, D. Assessment of postural stability in young healthy subjects based on directional features of posturographic data: Vision and gender effects. Acta Neurobiol. Exp. 2014, 74, 433-442.

17. Roemer, K.; Raisbeck, L. Temporal dependency of sway during single leg stance changes with age. Clin. Biomech. 2015, 30, 66-70. [CrossRef]

18. Huurnink, A.; Fransz, D.P.; Kingma, I.; de Boode, V.A.; van Dieën, J.H. The assessment of single-leg drop jump landing performance by means of ground reaction forces: A methodological study. Gait Posture 2019, 73, 80-85. [CrossRef]

19. Kozinc, Ž.; Löfler, S.; Hofer, C.; Carraro, U.; Šarabon, N. Diagnostic balance tests for assessing risk of falls and distinguishing older adult fallers and non-fallers: A systematic review with meta-analysis. Diagnostics 2020, 10, 667. [CrossRef]

20. Matsuda, S.; Demura, S.; Uchiyama, M. Centre of pressure sway characteristics during static one-legged stance of athletes from different sports. J. Sports Sci. 2008, 26, 775-779. [CrossRef]

21. Andreeva, A.; Melnikov, A.; Skvortsov, D.; Akhmerova, K.; Vavaev, A.; Golov, A.; Draugelite, V.; Nikolaev, R.; Chechelnickaia, S.; Zhuk, D. Postural Stability in Athletes: The Role of Age, Sex, Performance Level, and Athlete Shoe Features. Sports 2020, 8, 89. [CrossRef]

22. Riemann, B.L.; Schmitz, R. The relationship between various modes of single leg postural control assessment. Int. J. Sports Phys. Ther. 2012, 7, 257-266. [PubMed]

23. Nevitt, M.C.; Cummings, S.R.; Hudes, E.S. Risk Factors for Injurious Falls: A Prospective Study. J. Gerontol. 1991, 46, M164-M170. [CrossRef] [PubMed]

24. Paillard, T.; Noé, F.; Rivière, T.; Marion, V.; Montoya, R.; Dupui, P. Postural performance and strategy in the unipedal stance of soccer players at different levels of competition. J. Athl. Train. 2006, 41, 172-176. [PubMed]

25. Kozinc, Ž.; Žitnik, J.; Smajla, D.; Šarabon, N. The difference between squat jump and countermovement jump in 770 male and female participants from different sports. Eur. J. Sport Sci. 2021, 1-24. [CrossRef]

26. Kozinc, Ž.; Šarabon, N. Bilateral deficit in countermovement jump and its association with change of direction performance in basketball and tennis players. Sport. Biomech. 2021, 1-14. [CrossRef]

27. Sarabon, N.; Kern, H.; Loefler, S.; Jernej, R. Selection of body sway parameters according to their sensitivity and repeatability. Eur. J. Transl. Myol. 2010, 20, 5. [CrossRef]

28. Bakeman, R. Recommended effect size statistics for repeated measures designs. Behav. Res. Methods 2005, 37, 379-384. [CrossRef]

29. Hertel, J.; Olmsted-Kramer, L.C.; Challis, J.H. Time-to-boundary measures of postural control during single leg quiet standing. J. Appl. Biomech. 2006, 22, 67-73. [CrossRef]

30. Grace Gaerlan, M.; Alpert, P.T.; Cross, C.; Louis, M.; Kowalski, S. Postural balance in young adults: The role of visual, vestibular and somatosensory systems. J. Am. Acad. Nurse Pract. 2012, 24, 375-381. [CrossRef] 
31. Torres, S.F.; Reis, J.G.; de Abreu, D.C.C. Influence of gender and physical exercise on balance of healthy young adults. Fisioter. Mov. 2014, 27, 399-406. [CrossRef]

32. Nolan, L.; Grigorenko, A.; Thorstensson, A. Balance control: Sex and age differences in 9- to 16-year-olds. Dev. Med. Child Neurol. 2005, 47, 449-454. [CrossRef] [PubMed]

33. Duzgun, I.; Kanbur, N.O.; Baltaci, G.; Aydin, T. Effect of Tanner stage on proprioception accuracy. J. Foot Ankle Surg. 2011, 50, 11-15. [CrossRef] [PubMed]

34. Gobbi, G.; Galli, D.; Carubbi, C.; Pelosi, A.; Lillia, M.; Gatti, R.; Queirolo, V.; Costantino, C.; Vitale, M.; Saccavini, M. Assessment of body plantar pressure in elite athletes: An observational study. Sport Sci. Health 2013, 9, 13-18. [CrossRef]

35. Crotts, D.; Thompson, B.; Nahom, M.; Ryan, S.; Newton, R.A. Balance abilities of professional dancers on select balance tests. J. Orthop. Sport. Phys. Ther. 1996, 23, 12-17. [CrossRef] [PubMed]

36. Maki, B.E.; McIlroy, W.E. The role of limb movements in maintaining upright stance: The "change-in-support" strategy. Phys. Ther. 1997, 77, 488-507. [CrossRef]

37. Bruyneel, A.V.; Mesure, S.; Paré, J.C.; Bertrand, M. Organization of postural equilibrium in several planes in ballet dancers. Neurosci. Lett. 2010, 485, 228-232. [CrossRef]

38. Borzucka, D.; Kręcisz, K.; Rektor, Z.; Kuczyński, M. Postural control in top-level female volleyball players. BMC Sports Sci. Med. Rehabil. 2020, 12, 65. [CrossRef]

39. Horak, F.B.; Henry, S.M.; Shumway-Cook, A. Postural perturbations: New insights for treatment of balance disorders. Phys. Ther. 1997, 77, 517-533. [CrossRef]

40. Cheng, H.; Law, C.; Pan, H.; Hsiao, Y.; Hu, J.; Chuang, F.; Huang, M. Preliminary results of dancing exercise on postural stability in adolescent females. Kaohsiung J. Med. Sci. 2011, 27, 566-572. [CrossRef] 